\title{
ESTADOS INDEPENDIENTES: UNA PERSPECTIVA DESDE RELACIONES INTERNACIONALES
}

\author{
INDEPENDENT STATES: A PERSPECTIVE FROM \\ INTERNATIONAL RELATIONS
}

\section{Carlos Murillo Zamora ${ }^{1}$ \\ ORCID: : 0000-0001-5104-7675}

\section{RESUMEN}

El actor central en las relaciones internacionales es el Estado soberano independiente, según la naturaleza del sistema westfaliano; sin embargo, en la disciplina no se han desarrollado enfoques teóricos sobre el proceso de independencia. Se considera más un asunto del derecho internacional y de los intereses políticos de los actores estatales.

Por ello, se analiza en este trabajo, la cuestión de la independencia como un paso básico para que las entidades políticas accedan a la categoría de Estado, lo que sirve de fundamento para entender el papel del Estado independiente en el orden westfaliano. Al reconocer que hay cambios profundos en el sistema internacional, es necesario observar las variaciones en la concepción de independencia, sobre todo en relación con el principio de autodeterminación y el reconocimiento.

Esto tiene mayor relevancia a partir de la década de 1990, por los procesos de desintegración de algunos países, en particular, el caso de Kosovo; otra experiencia relevante es Taiwán. A lo anterior, se suma el debilitamiento del Estado, con la aparición de variantes que cuestionan la estatidad y la existencia del actor estatal. Al final, se hace una breve referencia a la experiencia latinoamericana y centroamericana, que muestran particularidades desde el siglo XIX.

Palabras clave: autodeterminación; Estados; independencia; reconocimiento; responsabilidad de proteger; soberanía.

\begin{abstract}
According to the nature of the Westphalian system, the independent state is the central actor in international relations; however, the discipline has not developed theoretical approaches regarding the independence process which is considered more a concern of the international law and the political interests of state actors. Then, in this article, the issue of independence is analyzed as a basic step for political entities to access the statehood becoming this a basis for understanding the role of the independent State in the Westphalian order. Acknowledging the existence of deep changes in the international system, it is necessary to observe the variations in the conception of independence, especially regarding self-determination and recognition principle. This principle has greater relevance since the 1990 s due to the disintegration processes of some countries, being Kosovo one of the main cases. Taiwan is also a relevant experience. Another key point is the weakening process of the state, with the appearance of variants that question the status and existence of the state actor. At the end of this paper, a brief reference is made to the Latin and Central American experience, which show particularities since the 19th century.
\end{abstract}

Keywords: States; independence; recognition; responsibility to protect; self-determination; sovereign.

1 Universidad de Costa Rica; Universidad Nacional de Costa Rica. Profesor catedrático. Doctor en Gobierno y Políticas Públicas (UCR) y Máster en Relaciones Internacionales (UNA). Correo electrónico: camuza@gmail.com 


\section{Introducción}

El esquema westfaliano se fundamenta en Estados soberanos; por ende, independientes de alguna autoridad superior y libres de subordinación en el sistema internacional. Sin embargo, en la disciplina de Relaciones Internacionales (RI) ${ }^{2}$ no existe un enfoque o planteamiento teórico que pueda concebirse como una "teoría de la independencia de los Estados". Por eso, en la mayoría de los libros de texto sobre RI no se menciona el concepto. ${ }^{3}$

Esto implica que para hacer referencia al proceso de independencia de comunidades que aspiran a ser reconocidos como miembros plenos del sistema de Estados, debe abordarse la cuestión desde lo doméstico y de lo externo, identificando aquellos elementos que conducen hacia la constitución de integrantes, teniendo en cuenta algunas consideraciones propias del derecho internacional; pero sin entrar en asuntos propiamente jurídicos. Así, el segundo propósito de este artículo no es un análisis jurídico de la independencia estatal.

Lo anterior, porque en algunos asuntos la cuestión resulta paradójica, por cuanto hay que considerar si realmente se trata de independencia o de interdependencia. Esto porque las entidades que aspiran a ser independientes requieren de otras para lograrlo. Y si bien, el concepto clave, en la lógica westfaliana, es la soberanía, esta cede ante la necesidad de reconocimiento y coexistencia en un sistema anárquico y de membresía en organizaciones intergubernamentales, que obligan a limitaciones en la libertad de acción y gestión. La soberanía supone que la declaración de independencia de un Estado le genera la condición de soberano, pero al mismo tiempo lo obliga a limitar algunas conductas.

Otra consideración necesaria, sobre todo con motivo del bicentenario de los Estados latinoamericanos, particularmente los centroamericanos, es acerca de la evolución de la noción de independencia y la forma en que se adquiere. Esto porque ha habido varias etapas en la incorporación de nuevos miembros en los últimos doscientos años de historia de las relaciones internacionales.

La interrogante orientadora del trabajo se plantea en términos de ¿cuál es el punto fundamental de la independencia, tanto desde la perspectiva doméstica,

2 En este trabajo uso lo acostumbrado en la mayoría de los casos, RI para hacer referencia a Relaciones Internacionales - como disciplina- y a las $\mathrm{r}$. i. para el caso de las relaciones internacionales como campo estudio y realidad que constituye el objeto de la disciplina.

3 Por ejemplo, en el libro de conceptos de T. Diez, I. Bode y A. Da Costa (2011), no se alude a independencia. Tampoco en libros de textos como Baylis y Smith (2005), o Goldstein (2001), para citar solo dos de muchos. En este último caso, se indica que en el sistema internacional los Estados tendrán total independencia e integridad territorial (Goldstein, 2001, p. 302). 
como de la internacional, y que se manifiesta de manera concreta en la noción de estatalidad (statehood), lo cual está relacionado con la construcción de Estado (statebuilding)?

No se puede obviar que, a pesar de los cuestionamientos, la noción de Estado continúa siendo central en las r. i. del siglo XXI. Lo que sí resulta cada vez más difícil es formular un concepto de Estado que sirva para identificar a todos los actores estatales. Y se debe tener en cuenta que se suma la tesis del Estadonación a partir de los Tratados de Westfalia (1648).

Noción que fue adoptada por la escuela realista como la esencia del actor internacional: un Estado soberano conformado por una nación, independiente y autónoma. Aunque, tal concepción predominante tiene debilidades, porque no hay un gran número de Estados-nación, esto en el sentido étnico de tribu o volk (alemán) (Loughlin, 2010, p. 200). Lo relevante es que el Estado adquiere un predominio en la dinámica internacional, permitiendo considerársele “...la más fundamental unidad del orden legal internacional, pero también está situado en la cima de la mayoría de las paradojas que distingue, y así enmarca, el derecho internacional" (French, 2013, p. 1).

Sin duda, esto se relaciona con la estatalidad, considerada, a partir de criterios jurídicos, como una aspiración y, en última instancia, con consideraciones políticas y diplomáticas, las cuales determinan el reconocimiento y aceptación de una entidad como Estado (cfr. French, 2013, p. 3)

Por eso al hablar de esta materia algunos conceptos claves por considerar son: sistema westfaliano, teoría constitutiva de la condición de Estado -basada en la consideración de Estado como persona-, y teoría declarativa de la condición de Estado (fundamentada en territorio definido, población permanente, gobierno y capacidad de establecer relaciones con otros actores estatales).

Tratando de abordar los distintos aspectos enunciados de la problemática, en el primer apartado de este artículo se hace una aproximación al fenómeno. Luego se abordar la cuestión del Estado como actor clave en el modelo westfaliano. Posteriormente, está la cuestión del derecho a la autodeterminación y el reconocimiento; un tema que se hizo más complejo con los casos de Kosovo y Taiwán.

Después, en la cuarta sección, los cambios en la noción de independencia son abordados, teniendo en cuenta la doctrina de la responsabilidad de proteger y el derecho de intervenir. Lo relativo a la extinción de los Estados y la pérdida de 
independencia se analiza en la sexta sección. Finalmente, de forma sintética, la experiencia de América Latina y de Centroamérica es discutida.

\section{Independencia: una aproximación}

Los procesos de independencia han estado estrechamente vinculados, desde el siglo XIX, con la descolonización, que se presenta como una revuelta contra Occidente; sin embargo, no es un fenómeno uniforme en todo el mundo, principalmente, porque el "nacionalismo anticolonial" tiene manifestaciones específicas en distintas comunidades.

En ese sentido, la independencia está relacionada con el derecho a la autodeterminación como acción contraria a la tesis del positivismo legal y el paternalismo europeo en el caso de pueblos no preparados para autogobernarse (Jackson, 2016, p. 20). Esto condujo a un replanteamiento de la relación de fuerzas entre el centro y la periferia, que dio lugar, de acuerdo con Buzan y Lawson (2015, p. 204), a dos ideas: i) las potencias coloniales tienen obligaciones sobre el bienestar de la población de los territorios; y ii) el creciente nacionalismo entendido como que si un pueblo tiene capacidad de autogobierno tiene el derecho a gobernarse.

En los procesos de descolonización, tanto a inicios del siglo XIX, como tras la II Guerra Mundial (IIGM), intervino lo relativo a construcción del Estado (state-building) y la transferencia de soberanía negativa (Jackson, 2016, p. 26). ${ }^{4}$ A lo que A. Basu (2012, p. 76), alude como una situación de interdependencia, porque tras la declaración se establece una relación entre el yo y el otro y una historia de asimilación; por consiguiente, se vincula a la educación, la cultura y la tradición, al igual que a la dominación.

Esto dio lugar a esquemas de patronazgo o relación clientelar entre actores desiguales, lo cual es una característica de la política internacional contemporánea; sin embargo, no es nuevo, porque históricamente, las superpotencias han basado su política exterior en la adquisición de "Estados clientes" (Ricardo, 2021, p. 1).

El fin de la II GM introdujo una nueva oleada de procesos de independencia, esta vez producto de la descolonización ante el debilitamiento de las potencias coloniales y un cambio en las reglas del juego internacional; pero

4 Entendida como el resultado de una declaración de independencia por un territorio colonial que queda comprometido a lazos de cooperación militar, económica y cultural que retiene la antigua metrópoli (Jackson, 2016, p. 28). 
complementada con factores como el crecimiento científico, tecnológico, económico y militar, junto con los recursos materiales y de poder que intervienen en la condición de Estado y sus relaciones (Luns, 1964, p. 2). Por consiguiente, la independencia se entiende como el paso para obtener soberanía reconocida por otros Estados y convertirse en miembros del sistema internacional. Pero no significa absoluta libertad para adoptar conductas contrarias a las normas internacionales.

Por el contrario, implica una interacción limitada en un orden anárquico, en el que predominan las relaciones de poder. Por esto, no todo Estado independiente tiene el mismo espacio de maniobra. La pregunta es por qué prácticamente, toda comunidad política aspira a ser independiente; elemento al que se hará referencia en la siguiente sección.

\section{Estado independiente: el actor clave en el esquema westfaliano}

Los tratados de paz de Westfalia (1648), marcaron el inicio de un nuevo orden internacional como "...un sistema en el cual los Estados se reconocieron mutuamente de forma precisa y solo como Estados, más allá de la fe [religiosa] de los distintos soberanos" (Lingua, 2021).

Esto convirtió al actor estatal en la pieza clave del sistema internacional, por encima de cualquier otro agente que ocupara alguna posición en el esquema mundial. Así el Estado se constituyó, a partir del siglo XVII, en un poder impersonal omnipotente, llegando a ser un importante objeto de análisis en el pensamiento político; sin embargo, la teoría moderna del Estado es una tarea pendiente para lograr una adecuada caracterización y conceptualización (Loughlin, 2010, p. 183). Desde la perspectiva de RI este es el actor por antonomasia y se los reconoce como iguales, aunque hay diferencias significativas entre ellos.

Entonces, al pensar en la independencia, la definición de qué es un Estado resulta básica. Incluso es un tema que ha preocupado desde la antigüedad. Por eso, P. Brown (1915), cita a Cicerón, quien lo definía como: "un cuerpo de hombres unidos con el propósito de promover su seguridad mutua y obtener ventaja por su fortaleza combinada" (p. 313); y complementa teóricamente como "una comunidad políticamente organizada que tiene el derecho de ser parte de la familia de naciones" y "el derecho a ser tratada como un Estado es independiente del reconocimiento" (Brown, 1915, p. 313). En ese sentido, consiste en "...un conjunto de instituciones cuyas funciones son control social y toma de 
decisiones autoritarias y procesos de implementación" y buscan autonomía de otros Estado (Gruhn, 2015, p. 353).

El Estado surge en el marco de la modernidad y está vinculado a las ideas de reclamos colectivos de la vida moderna, por lo que está relacionado a dilemas como el natural-convencional, emocional-racional, intimidad-distancia social (individualismo-colectividad), entonces “...el Estado expresa el carácter intrínsecamente relacional de lo individual y lo colectivo" (Loughlin, 2010, p. 202).

Así resulta de una creación europea de los siglos XV y XVI, que tiene lugar junto con el desarrollo del capitalismo, la ciencia y la filosofía moderna (Gruhn, 2015, p. 353). Pero surge como una entidad soberana y por tanto aislada, poseedora de fronteras impermeables que separan ámbitos políticos -por ende, "fronteras ontológicamente reales"- con recursos a la diplomacia y a la guerra, lo cual implica reafirmación de la nacionalidad como identidad básica para tener relaciones con otras comunidades (Fierke, 2017, p. 169-170). Pero al mismo tiempo, interactuando en un sistema que llegó a ser realmente mundial en el siglo $\mathrm{XX}$, con una creciente interdependencia.

Por supuesto, también hay aspectos epistemológicos. Hay facciones que consideran que el Estado es un hecho y aquellos que señalan que es una construcción legal; pero también divisiones entre subjetivistas y objetivistas. Para los primeros es una cuestión intersubjetiva en la definición de los criterios de la condición de Estado y la tesis de que es un fenómeno establecido en el derecho internacional (Aspremont, 2014, p. 204). Mientras que, desde la perspectiva ontológica, la cuestión es la condición previa de la entidad para ser reconocida como Estado, que genera la doctrina de los tres elementos de la Convención de Montevideo.

Así la idea de una "comunidad política eterna" vinculada al Estado-nación independiente es la base del sistema diplomático de los dos últimos siglos; por lo que se generó una construcción discursiva en torno a un "mundo civilizado" y una "ideología de progreso" que conducía a la ficción de una "comunidad política perfecta", la cual respondía al "derecho nacional a la autodeterminación" asentada en una "estructura ideacional" (Cornago, 2017, p. 328). En pocas palabras, una compleja construcción social que ha evolucionado de distintas formas y genera diversas estructuras estatales.

Ahora bien, es necesario considerar si una comunidad política posee lo esencial para operar como un Estado, por lo que se debe tener en cuenta: voluntad de reciprocidad, organización interna, territorio, comunidad de intereses y personalidad estatal (Brown, 1915, p. 321). 
Esto condujo a la Convención de Montevideo de 1933, en la cual un Estado requiere población permanente, territorio determinado, gobierno y capacidad de establecer relaciones diplomáticas con otros Estados (Art. 1); por lo que su existencia política no depende del reconocimiento de otros; así antes de tal acto “...tiene el derecho de defender su integridad e independencia, proveer a su conservación y prosperidad y, por consiguiente, de organizarse como mejor lo entendiere, legislar sobre sus intereses, administrar sus servicios y determinar la jurisdicción y competencia de sus tribunales" (Art. 3).

Esto hace que el reconocimiento constituya la aceptación del otro y es un acto incondicional e irrevocable (Art. 6). La cuestión, desde la perspectiva de esta convención, es que, si bien no se requiere del reconocimiento para ser Estado, solo ese acto de los otros es lo que le permite ser considerado miembro del sistema.

Pero también se trata de la estatalidad ya mencionada, que supone cuatro propiedades: i) capacidad de externalizar su poder y ser reconocida como unidad soberana; ii) capacidad de institucionalizar su autoridad que garantice el monopolio sobre los medios de coerción; iii) capacidad de diferenciar su control mediante un marco institucional particular y legítimo; y iv) capacidad de internalizar una identidad colectiva utilizando símbolos que refuerzan el sentimiento de pertenencia y solidaridad social; así se genera un Estado nacional (Oszlak, 1983, p. 532).

Entonces no existe un derecho a la independencia per se; es decir, no toda comunidad política puede demandar la independencia como un derecho inherente. Requiere cumplir con ciertos requisitos: i) existencia; ii) razones de conveniencia mutua y utilidad para establecer relaciones con otros; y iii) capacidad de independencia y existencia autónoma (Brown, 195, p. 325).

Por consiguiente, para que un Estado exista y sea reconocido como tal en el sistema internacional debe atender dos dimensiones: una externa, en la cual la entidad es independiente de otros actores y sociedades políticas, siendo soberana y autónoma, para ser parte del sistema internacional a través del reconocimiento; y otra interna, caracterizada por un gobierno, un sistema político, una sociedad que se reconoce como tal, territorio delimitado y recursos materiales para atender las necesidades de la población y la defensa.

Por lo que en criterio de R. Griffiths (2016, p. 3), la ruta menos problemática para la independencia es el consentimiento del Estado, eliminando el veto a la separación de una región; como en el pasado ocurrió con la descolonización (hoy quedan pocos casos de territorios coloniales o de ultramar); pero también hay una vía de disolución, como se interpretó el caso de Yugoslavia, y una 
cuarta concebida como un "derecho reparador de las naciones a la independencia" producto de las atrocidades cometidas en un Estado, como se insinúa ocurrió en el caso de Kosovo.

Tal y como he indicado, la soberanía, de naturaleza westfaliana, es el factor determinante en la independencia del Estado, pues es una representación de la autonomía de la esfera pública, "...un símbolo de la autoridad absoluta de esa esfera" (Loughlin, 2010, p. 186). Y mantiene una estrecha relación con la anarquía, porque se trata de actores independientes de autoridades exteriores, puesto que la "soberanía westfaliana" es absoluta e indivisible. Sin embargo, existen relaciones de patronazgo y eso significa que "...Estados soberanos con pequeñas capacidades deciden, 'voluntariamente', subordinarse a la autoridad de una Gran Potencia a cambio de recursos críticos para su reproducción social" (Ricardo, 2021, p. 3).

Para C. Reus-Smit (2011), la soberanía, y por ende la independencia, es un derecho que descansa sobre normas intersubjetivas que defienden al Estado, por lo que "...La independencia política de los Estados soberanos del mundo no descansa simplemente sobre las variadas capacidades materiales para defender su autonomía política e integridad territorial” (p. 211).

Por eso hay que considerar la posición jurídica internacional de los Estados, determinada por convenciones internacionales, tales como la de relaciones diplomáticas (1961), relaciones consulares (1963) y de relaciones entre Estados y organizaciones internacionales (1975). En ello, el punto medular es ¿cuál es la capacidad del actor internacional que asume ciertas competencias en el sistema internacional, que considera posee y le son reconocidas.

En este aspecto hay casos sui generis como la Santa Sede, al que se le reconoce el carácter de Estado en algunas organizaciones internacionales y mantiene relaciones diplomáticas con un importante número de Estados. ${ }^{5}$ Es un caso particular porque el objetivo de la diplomacia de la Santa Sede es esencialmente religioso, centrándose en un verdadero pacificador y no "trabajadores de la guerra o al menos trabajadores de los malentendidos” (Lingua, 2021).

5 En el caso de la Santa Sede hay que diferenciar entre el Estado, la parte geográfica o país: Ciudad del Vaticano, y la institución: Iglesia Católica. Esto tiene un excursus histórico prolongado. Al respecto G. Lingua (2021) anota que desde sus inicios “...la Iglesia y mucho antes del nacimiento de su poder temporal, es decir, de un Estado territorial, el Papa envió sus representantes para mantener contacto constante con las diferentes realidades eclesiales geográficamente distantes de Roma. El objetivo fue mantener la cristiandad unida bajo la soberanía espiritual del jefe de Estado de la Ciudad del Vaticano". 
Es evidente que los Estados, en la concepción westfaliana, resultan entidades complejas y diversas. Sin embargo, tal y como se indicó, parece que todas las comunidades políticas aspiran a serlo.

\section{Autodeterminación y reconocimiento: dos principios claves}

Desde la perspectiva del derecho internacional, un Estado puede tener poder para, pero no derecho a existir, pues "...existen como entidades separadas, autónomas, susceptibles de derechos y obligaciones"; aunque no pueden sobrevivir en forma aislada, requieren del contacto constante y son mutuamente dependientes, son teóricamente libres e independientes (Brown, 1915, p. 321-322).

Por eso, es por lo que antes se aludió a una paradoja entre independencia y dependencia, característica del modelo westfaliano. Al respecto Brown (1915), considera que "...la concepción de Estados absolutamente independientes entre sí, viviendo como si estuvieran en un estado de naturaleza ficticio, es un antagonismo con la concepción de una comunidad de naciones sometida voluntariamente a un código común de derecho internacional" (p. 324).

Así, la autodeterminación se convirtió, durante la descolonización, en el derecho a estar libre de un gobierno colonial; pero en la práctica se tornó en algo mucho más complejo, pues pasó a ser la vía para convertirse en un Estado independiente (Cornago, 2017, p. 331). Y a lo colonial se agregan casos de secesionismo, llegando a ser la secesión una de las múltiples caras del derecho a la autodeterminación, generándose una contradicción entre dos principios de derecho internacional: i) derecho a la autodeterminación; y ii) respeto por la integridad territorial de los Estados. Esto conduce a la autodeterminación externa en el caso de movimientos secesionistas anticoloniales y a la autodeterminación interna como derecho al autogobierno democrático a lo interno de un Estado (Cornago, 2017, pp. 332-333).

Por otra parte, el conjunto de normas regulando el proceso de reconocimiento está en crisis existencial, el cómo combinar aspectos legales y políticos, tras la desintegración de Yugoslavia y la consiguiente creación de nuevos Estados, ha generado problemas (Ryngaert \& Sobrie, 2011, p. 467). Sin embargo, la pregunta básica es si el reconocimiento es una condición esencial de la condición de Estado o la confirmación de una situación preexistente. Es el debate entre las escuelas constitutiva y declarativa. Para la primera, el Estado existe solo cuando es reconocido; por tanto, el reconocimiento es la conditio sine qua non y se 
fundamenta en el jus gentium voluntarium que señala que un Estado solo puede existir por el consenso de los otros Estados.

Entonces se cuestiona que “...cuánto reconocimiento de otros Estados es necesario antes que una entidad se 'transforme' en un Estado y si la decisión para reconocer estaría basada en hechos, normas, consideraciones geopolíticas o una combinación de factores" (Ryngaert \& Sobrie, 2011, p. 469).

La segunda escuela, la declarativa, considera que “...la condición de Estado está totalmente determinada por un conjunto de condiciones factuales, tales como una población permanente, un territorio fijo, un gobierno y la habilidad para mantener relaciones con otros Estados", por lo que cuando posee esos criterios entonces es un Estado erga omnes, así el reconocimiento es una formalidad que reconoce una situación factual y es "....un acto retroactivo que retorna al origen del momento en el que los criterios factuales fueron alcanzados y la entidad llegó a ser un Estado" (Ryngaert \& Sobrie, 2011, p. 470). Esta es hoy la teoría que predomina.

Señalan C. Ryngaert y S. Sobrie (2011), que una tercera vía argumenta que el reconocimiento no es ni constitutivo, ni declarativo, sino que se trata de una cuestión de efectividad, "...con el reconocimiento como un acto político que refuerza la efectividad internacional de una entidad", por lo que este es "... tanto constitutivo - dado que crea formalmente relaciones entre el Estado reconocedor y el reconocido-y declarativo -dado que, por sí mismo, la condición de Estado no se otorga en la entidad", lo cual no resuelve la brecha entre las dos principales escuelas (p. 471).

La cuestión de la autodeterminación y el reconocimiento, que desde finales de la Guerra Fría (GF) comenzó a ser cuestionada, adquirió una nueva dimensión con los casos de Kosovo, Osetia del Sur y Abjasia. En el primer caso, hubo una declaración unilateral de independencia, que condujo a que la Corte Internacional de Justicia emitiera una opinión consultiva en 2010, generando un escenario complejo, pues en el párrafo 56 de la opinión señaló “...es totalmente posible para un acto particular -tal como una declaración unilateral de independenciano constituir violación del derecho internacional sin necesariamente constituir el ejercicio de un derecho conferido por este” y agregó, par. 80, “...el alcance del principio de integridad territorial está confinado a la esfera de las relaciones entre Estados”. Y concluyó, par. 122, que “...la adopción de la declaración de independencia del 17 de febrero de 2008 no viola el derecho internacional general, la resolución 1244 (1999) del Consejo de Seguridad o el marco 
constitucional", entonces, reafirma que “...la adopción de esa declaración no viola alguna de las reglas aplicables del derecho internacional". 6

La cuestión con las declaraciones unilaterales es que generan conflicto con el principio de integridad territorial, que busca proteger al Estado original. En esta materia hay que considerar lo que establece la resolución de la Asamblea General de la ONU 2625/XXV de 1970 sobre la Declaración de principios de derecho internacional referentes a las relaciones de amistad y a la cooperación entre los Estados de conformidad con la Carta de las Naciones Unidas, a lo que se hará referencia más adelante.

En esta se reconoce la libre autodeterminación de los pueblos y que el territorio de una colonia se considera distinto del territorio del Estado que lo administra; sin embargo, el mismo texto dispone que ninguno de los principios se encamina a "...quebrantar o menoscabar, total o parcialmente, la integridad territorial de Estados soberanos e independientes que se conduzcan de conformidad con el principio de la igualdad de derechos y de la libre autodeterminación de los pueblos".

Por eso, J. Summers (2013, p. 229), considera que el derecho a la autodeterminación es de manera frustrante, ambiguo, pues el sujeto, "el pueblo", no está claramente definido, pudiendo ser usado en forma contradictoria, ya sea para dividir el Estado, proteger la soberanía o alentar la intervención.

Así, el caso de Kosovo plantea desafíos al derecho a la autodeterminación, tales como: ¿Qué pueblos tienen ese derecho? ¿Cómo el derecho se traduce en soberanía y categoría de Estado? ¿Cómo es reconocida la categoría de Estado soberano? ¿Cuándo la intervención humanitaria es legítima? (Economides, 2013, p. 823).

Esto se debe contextualizar en el marco de los cambios sistémicos tras el fin de la GF, que generó una "fase transformacional", con expresiones como la guerra en el Golfo Pérsico, el colapso estatal de Somalia, genocidio en Ruanda y desintegración violenta de Yugoslavia, a lo que suma el 9-11, las intervenciones en Irak y Afganistán y los conflictos en la República Democrática del Congo, Sierra Leona, Sudán, Georgia, Libia y Siria (Economides, 2013, pp. 823-824). Ello incrementó las complejidades y la heterogeneidad de los Estados independientes.

6 International Court of Justice, Kosovo Advisory Opinion of July 22, 2010. https://www.icj-cij.org/en/ case $/ 141$ 


\section{Cambios en la noción de independencia en el siglo XXI}

Tras el fin de la GF y la ruptura del orden internacional de la segunda mitad del siglo XX, surge no solo una nueva arquitectura sistémica, sino cambios profundos en el esquema westfaliano. Así, múltiples factores, tales como regímenes legales, relaciones de poder y legitimidades en competencia, en los niveles local, nacional, regional e internacional, han moldeado o inhibido los procesos de independencia (Fierke, 2017, p. 168). Esto puso sobre la mesa cuestiones como ¿qué significa la independencia en la práctica? Ello tiene connotaciones ontológicas que aún no han sido clarificadas. En este sentido, el sistema internacional hoy es claramente poswestfaliano.

Y en tal situación, la soberanía constituye un problema para la noción de independencia. Hay contradicciones entre ellas; la soberanía westfaliana es propia del siglo XVI; mientras que la noción de independencia se ubica a partir de mediados del siglo XX y está vinculada a la soberanía popular o nacional.

Incluso, en el siglo XIX, bajo la concepción de la Declaración de Independencia de Estados Unidos (EUA), la idea de soberanía popular está basada en la capacidad de la sociedad para definir su forma de gobierno independiente de la influencia externa; y en el ámbito internacional se entiende como un sistema que reconoce la existencia autónoma del Estado soberano. Bajo tal arquitectura internacional, los Estados mantienen relaciones bajo el principio de no interferencia en asuntos domésticos y se tratan como iguales (cfr. Fierke, 2017, p. 171).

Entonces se genera la cuestión compleja que ya se mencionó, porque se trata de soberanía, independencia, nacionalidad, territorio (esto implica fronteras con otras unidades estatales) e identidad étnica o de nación. Pensar esto en términos westfalianos no tiene mucha lógica en el siglo XXI, pues existen otros criterios para la construcción de Estado (statebuilding) y las relaciones entre unidades estatales.

Se trata de una mezcla entre modernidad y posmodernidad, concebidos en términos de cosmovisiones como la eurocéntrica y sinocéntrica, junto con historias enredadas y multivectoriales, lo cual tiene lugar en un escenario de transformaciones globales, que originan una nueva arquitectura sistémica y a la reconceptualización de las relaciones internacionales (Murillo, 2018 y 2020).

A esto se suman nuevos proyectos hegemónicos, como el chino (de naturaleza confuciana), que superan las nociones westfalianas básicas y complementan los 
cambios por la revolución en las tecnologías de información, comunicación y las dinámicas del ciberespacio.

Hoy operan varias premisas ontológicas. Por una parte, el mundo continúa siendo un sistema dividido en espacios territoriales definidos por fronteras -cada vez más porosas-, caracterizado por las interacciones de conflicto y cooperación entre agencias claramente diferenciadas (unidades estatales), asentadas en la noción mecanicista de balance de poder; mientras que, por otra, se alude a la identidad fundamentada en cosmovisiones y concepciones civilizacionales, como las que impulsan Nueva Delhi y Pekín, que integran elementos identitarios, étnicos y filosóficos, los cuales rompen con la tradición westfaliana, incorporando nociones como la gobernanza global (en el caso de China se alude a Tianxia y las tesis confucianas) (Fierke, 2017, pp. 174-175)7.

Tal dinámica ha creado un escenario en el que persiste el caos hobbesiano, porque no hay una sociedad mundial coherente con instituciones políticas universalmente aceptadas; no hay una identidad política global. Pero todas las comunidades aspiran a ingresar a ese escenario, esperando que cambie a un esquema menos caótico como el grociano.

Lo anterior lleva a M. Riegl y B. Dobos (2018, pp. 443-444), a considerar que se ha desafiado el principio básico del orden westfaliano de Estado-no Estado, que corresponde a la naturaleza geopolítica y la calidad de condición de Estado, pues han aparecido entidades que quedan a medio camino en ese modelo binario y resultan en un "Estado de facto", incluso con entidades etiquetadas de ilegales, patológicas y clandestinas, que no alcanzan el control territorial propio de los Estados, lo cual favorece los intentos secesionistas, tales como Eritrea, Timor Oriental y Sudán del Sur, o casos más complejos como Palestina y Sahara Occidental, al igual que entidades con reconocimiento e independencia de facto como Taiwán, Kosovo y Abjasia.

Mientras que la separación de Yugoslavia introdujo variaciones significativas y la aparición de nuevas normas, incluidas las de la Comisión Europea sobre el reconocimiento de las nuevas repúblicas, en particular, con la Comisión Badinter, que abordó aspectos legales como soberanía, reconocimiento, derecho a la autodeterminación y sucesión estatal (Ryngaert \& Sobrie, 2011, p. 475).

7 Sobre la noción china de "todo bajo el cielo" o Tian-xia, Zhao (2009, p. 9) sugiere que se trata un esquema de subestados autónomos que coexisten en un Estado global, pero contribuyen a acuerdos universales y un esquema de instituciones globales que aseguran el orden mundial; por lo que el mundo se concibe como uno físico (tierra), uno sicológico (sentimiento de la gente) y uno institucional (institución mundial). Entonces como lo entiende la filosofía china: nada puede ser definido si no se tienen en cuenta las relaciones, porque las cosas dependen de sus vinculaciones (Zhao, 2009, p. 9). 
Taiwán es un caso particular, pues mantiene relaciones diplomáticas con unos 14 Estados e incluso, en su carácter de República de China, ocupó el puesto permanente en el Consejo de Seguridad. Además, tiene oficinas consulares y comerciales en un gran número de países, incluidos Estados Unidos y la mayoría de los europeos. En este sentido, tiene una condición estatalidad mayor a la de muchos miembros de Naciones Unidas.

Por eso, no se puede dejar de lado el rol de las superpotencias y grandes potencias. Porque no es lo mismo que un grupo de países pequeños reconozcan a una entidad estatal como independiente, que lo haga una superpotencia. Esto es importante cuando se reconoce que China y Rusia (que se apegó al esquema westfaliano bajo la bandera de la URSS) utilizan otras vías de intervención, cuestionando los valores occidentales y actuando en una vía bilateral y diplomática con apoyo de recursos coercitivos.

Hoy el mundo es universal, multicultural y multirregional, aunque con predominio de Estados soberanos, que coexisten con otros en decadencia; es muy diferente al del siglo XVII, que estaba confinado a Europa y dominado por la visión de la cristiandad. Para tal cambio, fueron necesarias -en criterio de C. Reus-Smit (2011, p. 208) - cinco oleadas de globalización: el modelo westfaliano, la independencia de América Latina, el tratado de Versalles de 1919, la descolonización posterior a 1945 y el colapso del bloque soviético. A estas se podría sumar la aceleración de la globalización producida por los atentados del 9-11 y los que le sucedieron en Europa.

En RI esas oleadas son explicadas de distinta forma por las diferentes teorías (aspecto que no se profundizará en este artículo); mientras que Reus-Smith (2011, p. 208), favorece la importancia de las luchas populares por el reconocimiento de los derechos individuales, como el mecanismo para la expansión del sistema internacional. En el entendido que el Estado independiente continuará siendo el actor dominante.

\section{Extinción de los Estados: ¿pérdida de independencia?}

Cada vez más aparecen conceptos sobre la decadencia del Estado, que hacen pensar en una entidad en crisis y hasta obsoleta; principalmente, porque los fundamentos están en la idea del estándar de Estado-nación europea, cuando hoy existen diversas estructuras estatales (King \& Le Galès, 2012, p. 109). Por lo que hay múltiples dicotomías, como entre fuertes y débiles y los fallidos en sus distintas versiones; pues la dinámica sistémica influye de distinta forma en 
los Estados que aparecieron en las últimas décadas y los del siglo XIX (King \& Le Galès, 2012, p. 110).

Ello hace que hoy haya Estados independientes enfrentados con escenarios de violencia estructural y sistémica; y estructuras estatales desmanteladas. También, hay gran variedad en los indicadores de la condición de Estados, por lo que resulta en una figura heterogénea y difusa, al igual que su análisis.

Asimismo, cada vez hay menos consenso sobre los factores esenciales de un Estado. Sin embargo, tiende a haber un predominio de la tradición weberiana en torno al monopolio de la violencia y la concentración de los recursos coercitivos, por la capacidad administrativa para conducir y gobernar una sociedad, establecer normas, garantizar los intercambios, gestionar los impuestos y organizar el desarrollo económico, así como proteger a la ciudadanía (King \& Le Galès, 2012, p. 116).

Sin embargo, la existencia de Estados frágiles genera un problema para la idea de "Estados soberanos e iguales". Por ejemplo, el G7 y OCDE (Organización para la Cooperación y el Desarrollo Económicos) impulsan el International Dialogue on Peacebuilding and Statebuilding. ${ }^{8}$ Pero esto contradice la idea de soberanía westfaliana y la de Estados autónomos e independientes.

En la práctica, los Estados débiles se vieron favorecidos con ese esquema, porque participaban en espacios internacionales a pesar de la falta de capacidad gubernamental y durante la GF el rol de las superpotencias los apoyó; pero esto cambio tras el fin de ese periodo (Pospisil, 2017, p. 1419). Detrás de estos asuntos está el cómo se entendió el derecho de autodeterminación y el reconocimiento en el mundo bipolar.

En 2001, el reporte de la "Responsabilidad de Proteger", producto del trabajo de la Comisión Internacional sobre Intervención y Soberanía Estatal, redefinió algunos principios de la soberanía, por lo que esta dejó de ser "... .una protección contra la interferencia externa, para ser una 'responsabilidad compartida' que tiene que ser constantemente demostrada por una conducta cumpliendo las expectativas de las potencias globales dominantes" entonces "el intervencionismo humanitario y la construcción internacional de Estado conduce, así, a un replanteamiento de los entendimientos tradicionales de la soberanía", por lo que "la soberanía llega a ser compartida" (Pospisil, 2017, p. 1420).

8 Es un foro sobre diálogo político para atender a los países afectados por conflictos y fragilidad, apoyado por distintos grupos y vinculado con la iniciativa The New Deal for Engagement in Fragile States; sus orígenes están en la Declaración de Paris sobre efectividad de la ayuda al desarrollo (2005) y se estableció en 2008. 
Es evidente que desde la década de 1990 la soberanía pierde la condición de atributo fijo de la categoría de Estado, para convertirse en un atributo contingente y dependiente sobre percepciones y prácticas (Pospisil, 2017, p. 1421). La cuestión es hasta dónde llegará el proceso de decadencia del Estado, sin que pierda sentido considerar que es el actor predominante.

\section{América Latina y Centroamérica: algunas sugerencias}

En el siglo XIX, las nuevas repúblicas en América Latina surgen de jurisdicciones internas preestablecidas, que constituían posesiones bajo la administración de un virrey o capitán, por lo que se generó un periodo de convulsiones internas y de complejas relaciones externas, sobre todo con la Corona Española y las potencias coloniales europeas.

Sin embargo, los virreinatos y capitanías no lograron convertirse en unidades estatales, sino que se fragmentaron, al mismo tiempo que pasaron de la noción de ius gentium al concepto de ius inter gentes (Cornago, 2017, p. 343). Por lo que surgió una diplomacia provincial conducente a nuevos Estados, que según Gutiérrez (citado en Cornago, 2017): "El principal objetivo de esta diplomacia provincial o constitutiva era remediar la disolución de su unidad más amplia y restablecer los lazos sociales que habían sido aplastados con la deposición de las autoridades virreinales" (p. 343). Ese fenómeno fue exclusivo de los territorios coloniales españoles.

Centroamérica no fue la excepción, por lo que se produjeron varios intentos por establecer un esquema federal, o incluso adhesión a las iniciativas mexicanas. Sin embargo, a mediados del siglo XIX, consideraban la principal amenaza a su soberanía a sus vecinos, más que a las potencias europeas y a EUA. Aunque una amenaza sí provino de Washington con los proyectos colonialistas y económicos para establecer enclaves como el de la zona de Chiriquí (Harris, 2020, p. 2).

Así el istmo centroamericano se debatió entre nacionalismo y regionalismo. A lo que se sumó la agitación anticolonialista, frente a las iniciativas de Lincoln y las políticas británicas (Harris, 2020, p. 7). Aunque hubo posiciones distintas en cada uno de los países, pues Costa Rica mostró la posibilidad de aceptar a inmigrantes esclavos liberados en EUA para repoblar la zona sureste del país -territorio en disputa con Nueva Granada- (Harris, 2020, p. 8). 
Sin embargo, eso no significaba que aceptara, al igual que los otros países centroamericanos, una colonia en el Istmo (Harris, 2020, p. 11). En resumen, a mediados de siglo XIX, las principales amenazas a la soberanía de los países centroamericanos fueron EUA y Gran Bretaña, quienes buscaban un punto de apoyo en el Istmo con fines geográficos, políticos y económicos (Harris, 2020, p. 22).

De esta manera se podría decir que América Latina y Centroamérica constituyeron una experiencia de formación de Estado y estatalidad particulares. Esto no ha sido apropiadamente contextualizado en el sistema internacional, en parte por las concepciones que se tienen en la región de lo que es ser un Estado independiente.

Tal característica es lo que conduce a que se aluda a una "segunda independencia”, que supere las causas inconclusas de la primera (Pinedo, 2010, p. 173). La cuestión es si, con motivo del bicentenario, tiene sentido pensar en esos términos: o más bien se trata de un problema de construcción del Estado y la estatalidad, más que de independencia.

\section{Consideraciones finales}

Los Estados independientes, caracterizados por la soberanía westfaliana, son la pieza central del sistema internacional, los actores por antonomasia y se conciben como actores que no están subordinados a una autoridad superior y se reconocen como iguales; mientras que a lo interno tienen autonomía para decidir sobre su sistema político y organización. Poseen una frontera que claramente separa los ámbitos doméstico y externo.

A pesar de esa relevancia para las r. i., lo cierto es que en RI no hay una teoría o enfoque que explique el proceso mediante el cual una entidad política se declara independiente y asume la categoría de Estado, para llegar a ser miembro del sistema a través del reconocimiento. Generalmente, se le considera un asunto del derecho internacional o de los intereses y las acciones propias de cada gobierno.

Durante el siglo XIX y la primera mitad del XX, la gran mayoría de nuevos Estados resultaron del proceso de descolonización, aunque con diferencias entre los de la primera de esas centurias, que correspondieron -en gran parte- a territorios bajo dominio de la Corona Española, y los que se independizaron tras el fin de la IIGM. Mientras que durante la GF a los territorios coloniales se sumaron algunas secesiones, pero en todo momento bajo la tutela de alguna de las superpotencias. 
La situación cambia, en forma radical, con el fin del orden bipolar de posguerra mundial, sobre todo con la desintegración de Yugoslavia y del bloque soviético en la década de 1990. Esto introdujo variantes en los constructos y las prácticas, al mismo tiempo que generó problemas en la concepción de la autodeterminación y el reconocimiento. Un factor adicional es la declaración unilateral de independencia. Kosovo ha sido el principal caso en esta materia.

Sin embargo, la concepción de independencia no conduce a criterios estandarizados y continúa siendo esencialmente un asunto político. Esto se comprueba con el caso de Taiwán, que posee un sólido proceso de construcción de Estado y un alto grado de estatalidad, pero que por la presión de China se impide que se le reconozca como miembro pleno del sistema internacional, como lo fue en el pasado. Así Taiwán es un actor internacional de facto, no de iure.

La adopción de doctrinas como la responsabilidad de proteger y el derecho a intervenir constituyen un cuestionamiento a la independencia de los Estados soberanos. De igual forma sucede con el debilitamiento de la estatalidad en un número cada vez mayor de Estados. Esto conduce a cuestionar la vigencia de una noción de independencia característica del esquema westfaliano.

Lo que sí resulta claro es que el concepto de Estado westfaliano independiente perdió vigencia en el siglo XXI; sin embargo, las entidades políticas no estatales mantienen sus aspiraciones para ser independientes e integrarse al concierto de Estados, más allá de cuál sea la solidez de su construcción y estatalidad. Por eso es necesario, para entender la dinámica de las $r$. i. en la actual coyuntura, comprender qué y cómo opera el proceso de independencia en un contexto muy diferente al de los dos siglos anteriores.

\section{Referencias}

Aspremont, J. (2014). The International law of statehood: craftsman for the elucidation and regulation of birth and death in the international society. Amsterdam Law School Legal Studies Research Paper 2014-37. https://hdl.handle.net/11245/1.445404

Basu, A. (2012). Postcolonialism: Independence or Interdependence?. The IUP Journal of English Studies, 7(3): 74-80. http://ebookcentral.proquest.com

Baylis, J., \& Smith, S. (2005). The Globalization of World Politics. An introduction to international relations ( $3^{\text {rd }}$ edition). Oxford University Press. 
Brown, P. (1915). The Theory of the Independence and Equality of States. The American Journal of International Law, 9(2): 305-335. https://www.jstor.org/ stable/2187161

Buzan, B. \& Lawson, G. (2015). The Global Transformation. History, Modernity and the Making of International Relations. Cambridge University Press.

Cornago, N. (2017). Beyond self-determination: norms contestation, constituent diplomacies and the co-production of sovereignty. Global Constitutionalism, 6(2): 327-358. https://doi.org/10.1017/S2045381717000132

Diez, T., Bode, I, \& Da Costa, A. (2011). Key Concepts in International Relations. Sage Publications. EBSCO Publishing AN: 372452.

Economides, S. (2013). Kosovo, Self-Determination and the International Order. Europe-Asia Studies, 65(5): 823-836. http://dx.doi.org/10.1080/09668136.20 13.802130

Fierke, K. (2017). Introduction: Independence, global entanglement, and the coproduction of sovereignty. Global Constitutionalism, 6(2): 167-183. https:// doi.org/10.1017/S2045381717000089

French, D. (2013). Introduction. In D. French (Ed.), Statehood and Self-Determination: Reconciling Tradition and Modernity in International Law (pp. 1-20). Cambridge: Cambridge University Press. https://doi.org/10.1017/ CBO9781139248952.003

Goldstein, J. (2001). International Relations ( $4^{\text {th }}$ edition). Longman.

Griffiths, R. (2016). The State of Secession in International Politics. E-International Relations. https://www.e-ir.info/2016/09/23/ the-state-of-secession-in-international-politics/

Gruhn, I. (2015). State Formation, Theory of. In N. Smelser \& P. Baltes (Eds.), International Encyclopedia of the Social \& Behavioral Science (2 ed), vol. 23. Cambridge University Press. http://dx.doi.org/10.1016/ B978-0-08-097086-8.93132-5

Harris, M. (2020). Struggle for Sovereignty: An African-American Colonization Attempt and Delicate Independence in Mid-Nineteenth Century Central America. Graduate Theses, Dissertations and Problem Report. West Virginia University. https://researchrepository.wvu.edu/etd/7600

Jackson, R. (2016). Independence by Right. In T. Young (Ed.), Readings in the International Relations of Africa (pp. 19-32). Indiana University Press. http:// ebookcentral.proquest.com 
King, D., \& Le Galès, P. (2012). State. In E. Amenta, K. Nash \& A. Scott (Eds.), The Wiley-Blackwell Companion to Political Sociology, pp 107-119. https:// doi.org/10.1002/9781444355093.ch10

Lingua, G. (2021, June 10). The juridical personality of the Holy See and its activity in the international community. Apostolska Nuncijatura U RH. Retrieved June 12, 2021, from https://ika.hkm.hr/apostolska-nuncijatura-u-rh/presentation-of-the-apostolic-nuncio-in-croatia-msgr-lingua-to-the-diplomatic-club/

Loughlin, M. (2010). Foundations of Public Law. Oxford University Press. http:// ebookcentral.proquest.com/

Luns, J. (1964). Independence or Interdependence. International Affairs, 40(1): 1-10. https://www.jstor.org/stable/2609693

Murillo, C. (2018). Reconceptualización de Relaciones Internacionales en un mundo transformado. Escuela de Relaciones Internacionales, Universidad Nacional, Costa Rica.

Murillo, C. (2020). Un siglo de Relaciones Internacionales: la necesidad de una reconceptualización en el marco del siglo XXI. Revista de Relaciones Internacionales de la UNAM. Núm. 138, septiembre-diciembre.

Oszlak, O. (1983). Reflexiones sobre la formación del Estado y la construcción de la sociedad argentina. Desarrollo Económico Revista de Ciencias Sociales. 21(84): 531-548. https://doi.org/10.2307/3466613

Pinedo, J. (2010). El concepto Segunda Independencia en la historia de las ideas en América Latina: una mirada desde el bicentenario. Atenea. 502: 151-177. http://dx.doi.org/10.4067/S0718-04622010000200009

Pospisil, J. (2017). 'Unsharing' sovereingty: G7+ and the politics of international statebuilding. International Affairs, 93(6): 1417-1434. https://doi.org/10.1093/ ia/iix 188

Reus-Smit, C. (2011). Struggles for Individual Rights and the Expansion of the International System. International Organization, 65(2); 207-242. https:// www.jstor.org/stable/23016811

Ricardo, R. (2021). Interstate Patronage and the Westphalian Narrative of International Politics. Academia Letters, Article 366. https://doi.org/10.20935/ AL366

Riegl, M., \& Dobos, B. (2018). Power and Recognition: How (Super)Powers Decide the International Recognition Process. Politics \& Policy, 46(3): 442-471. https://doi.org/10.1111/polp.12257 
Ryngaert, C., \& Sobrie, S. (2011). Recognition of States: International Law or Realpolitik? The Practice of Recognition in the Wake of Kosovo, South Osetia, and Abkhazia. Leiden Journal of International Law, 24(2): 467-490. https:// doi.org/10.1017/S0922156511000100

Summers, J. (2013). The internal and external aspects of self-determination reconsidered. In D. French (Ed.), Statehood and Self-Determination: Reconciling Tradition and Modernity in International Law (pp. 229-249). Cambridge: Cambridge University Press. https://doi.org/10.1017/CBO9781139248952.014

Zhao, T. (2009). A Political World Philosophy in terms of All-under-heaven (Tianxia). Diogenes, 221: 5-18. https://doi.org/10.1177/039219210910214 
